\title{
An Overview of Point-of-Care Testing Service
}

\author{
ASM Giasuddin ${ }^{1 *}$, KA Jhuma ${ }^{2}$ and AMM Haq ${ }^{3}$ \\ ${ }^{1}$ Professor of Biochemistry \& Immunology, Director MRU, MHWT, Mew\& H Building, Dhaka, Bangladesh \\ ${ }^{2} M R U, M H W T$ \& Department of Biochemistry, Medical College for Women \& Hospital, Dhaka, Bangladesh \\ ${ }^{3}$ MRU, MHWT \& Dept of Medicine, Medical College for Women \& Hospital, Dhaka, Bangladesh
}

*Corresponding author: ASM Giasuddin, Professor of Biochemistry \& Immunology \& Director, Medical Research Unit (MRU), MHWT, Medical College for Women \& Hospital Building, Uttara Model Town, Dhaka-1230, Bangladesh

Submission: 眥 May 24, 2018; Published: 眥 May 31, 2018

\begin{abstract}
Point-of-Care Testing (POCT) has become extremely important in diagnostic laboratory services confirming a clinical suspicion, i.e. including (ruling in) or excluding (ruling out) a clinical diagnosis. This is particularly true in setting such as emergency room, intensive care unit, casualty room, etc. The Royal college of Pathologists, UK and The institute of Biomedical Science, UK produced some POCT guidelines so that internal quality control (IQC) and external quality assessment (EQA) have been maintained ensuring the accuracy of results and therefore, avoiding and reducing in patients misdiagnosis. New emerging devices utilizing molecular techniques based on polymerase chain reaction (PCR) to provide POCT service for infectious diseases are likely to reach the commercial market in the next few years. The various facets of POCT i.e. technological requirements, POCT service implementation and management, POCT devices availability, IQC and EQA performance were discussed.
\end{abstract}

Keywords: Point-of-care; Point-of-care testing; Poet

\section{Introduction}

Diagnostic laboratory service should be given utmost importance and priority in confirming a clinical suspicion, i.e. including (ruling in) or excluding (ruling out) a clinical diagnosis. In this respect, Point-of-Care Testing (POCT) is extremely important in case of emergency medical/health care particularly. It is a diagnostic laboratory testing service performed in a member of different clinical settings such as emergency room, admission unit, operating room, intensive care unit (ICU), outpatient clinic, ward, etc. [1-3]. The evolution of small portable POCT devices/instruments that measure blood gases, electrolytes and other emergency analyses has helped immensely to set up POCT facilities. In many hospitals, POCT has become an integral part of diagnostic services, thus saving lives in ICU, casualties and emergencies specially $[2,4]$. POCT is an important part of Laboratory Medicine which includes diagnostics, teaching, research, administration, etc. However, analytical and technological requirements in term of instrumentation, manpower of heterogeneous categories i.e. laboratory consultants, biomedical scientists, medical technologists, others, logistics, etc must be considered carefully.

\section{Technological Requirement}

POCT introduces a range of issues such as management and visibility of remote testing site, data collection, quality control regime, analyzer status and the use of the equipment, analysis by certified personnel, resources required to deliver an extended diagnostic service while maintaining the level of quality which are to be considered adequately [1,2]. The Royal College of Pathologists, UK in 2004 stated that "a modern pathology service will need to consider a wide range of technologies to provide its service, and this may be achieved by linking disseminated laboratory services as part of networked area with information technology (IT) connection (e.g. by linking POCT systems remotely with central core laboratories)" [2,4]. A fully functional POCT connectivity network could also address the points raised in the POCT guidelines produced by the Institute of Biomedical Science, UK which indicates that "It is imperative that wherever POCT is operated that it is monitored and supervised by qualified staff of a Clinical Pathology Accreditation (CPA) accredited clinical laboratory" [5,6].

\section{POCT device availability}

The evolving technological advances that enabled the development of novel POCT instruments and the underlying analytical techniques are glucose biosensor strips and lateral flow strips using immobilized antibodies to determine a range of parameters including cardiac markers and infectious pathogens. The second category of devices is larger, often bench-top devices which are essentially laboratory instruments being reduced in both size and complexity. These include critical care unit analyzers and, more recently, small hematology and immunology analysers $[6,7]$. New emerging devices include those that are utilizing molecular 
techniques such as polymerase chain reaction (PCR) to provide infectious disease testing in a sufficiently small device to be used at the point of care. This area is likely to grow with many devices being developed and likely to reach the commercial market in the next few years [7]. Additionally, it is very desirable to measure various parameters simultaneously in the same specimen, allowing a rapid, low-cost, and reliable quantification. Therefore, multiplexed pointof-care testing (xPOCT) has become more important for medical diagnostics in the last decade. POCT has become established worldwide and finds vital roles also in public health [8].

\section{POCT service: implementation and management}

Originally most POCT tests were undertaken on patients who were acutely unwell, when rapid test results were needed for clinical reasons. However, in the recent past most POCT implementations were undertaken helping to ensure that patient pathways work more efficiently. For example, where patients arrive early in the morning and need blood tests before a procedure, the best way to ensure results are ready is via POCT. Even though POCT is more expensive on a cost-per test basis, the upstream and downstream savings it can provide mean that it does offer an economic saving overall.

The Ontario Laboratory Accreditation Body found that POCT had the highest error rate in comparison to other sources of error in the laboratory, highlighting the risk of error in Poet and the need for quality control $[9,10]$. Quality control comprises two key elements, internal quality control (IQC) and external quality assessment (EQA), both of which are already integral components of the laboratory quality system, IQC \& EQA and are designed to ensure that the quality of test results will not compromise the clinical care of the patient. IQC involves running samples containing analytes of a known concentration, to monitor the accuracy and precision of the analytical process over time. Frequency of testing will depend on the design of the device and risk of harm to the patient. When choosing an appropriate material performing quality control on POCT devices, it is important to look for materials that offer the following benefits: ease of use, a matrix similar to the patient sample, contains analytes at clinically relevant concentration, accurately assigned target values, from a third-party source offering an unbiased and independent assessment of analytical performance $[9,10]$.

EQA is also strongly recommended for POC devices for quality and competence relating to POCT in hospitals, clinical and ambulatory care environments. An EQA scheme assesses the accuracy of the POCT devices through direct results comparison of one device to identical devices worldwide. This peer comparison allows for assessment of the accuracy of a device over time and provides confidence that the patient results a device is reporting are accurate. There are many EQA schemes available for POCT devices. When choosing a scheme it is important that a POCT coordination team considers the following: Frequent reporting to minimize the amount of time an error can go unnoticed, quality material provided in a format suitable for use with POCT devices, well-designed reports that allow quick and easy trouble shooting of erroneous results at a glance, international scheme with a large number of participants to ensure a more accurate reflection of performance [10]. Interlaboratory data management software for ICQ \& EQA can allow a laboratory to manage and interpret quality control data. Such software can ensure that accuracy and precision are monitored over time, comparing results to POCT users worldwide and providing a true reflection of performance of both the device and the personnel using the device. The risk of harm to the patient while using a POCT device can only be reduced when there is a stringent quality control strategy in place and also ensuring the accuracy of results and therefore, a reduction in patient misdiagnosis [10].

\section{Conclusion}

POCT has become established worldwide and finds vital roles also in public health. The main advantages and potential operational benefits of it are reducing time for clinical decision making, operating time, high-dependence, postoperative care time, emergency room time, number of hospital beds required, risk of results being lost and ensuring optimal use of professional time and finally improved health outcomes. POCT has been shown to offer benefits for the patients and the economy of the health service systems. However, these benefits must always be weighed against the risk of patient harm. POCT will offer benefits only if the results obtained are accurate and reliable which makes quality control policies a vital consideration. Although there is no universal quality control strategy in place for POCT devices currently, it is well understood that IQC and EQA should be applied to POCT devices strictly. With more and more understanding on the biochemical and molecular basis of illnesses, the discipline of Medical Biochemistry played in the past, is playing presently and will play in the future as well increasingly important roles in the diagnosis, management and prevention of various human diseases worldwide. More recent developments in molecular techniques i.e. PCR through Medical Molecular Biology will have tremendous impact on Poet service.

\section{References}

1. Price CP, John AS (2015) Point-of- Care instrumentation. In: Burtis CA, Bruns DE (Eds.), Tietz Fundamentals of Clinical Chemistry and Molecular Diagnostics, ( $7^{\text {th }}$ edn), Elsevier Saunders, St. Louis, USA, pp. 272-285.

2. https://www.rcpath.org

3. Giasuddin ASM (2017) Point-of-care testing: Importance in emergency medical/health care (editorial). Bangladesh J Med Biochem 10(2): 4344.

4. Sladen A (2009) Point of care testing and IT connectivity. Point of Care Testing.

5. http://www.homerton.nhs.uk/media/440840/ibms-professionalguidance-poct.pdf

6. https://en.wikipedia.org/wiki/Point-of-care_testing

7. St John A, Price CP (2014) Existing and emerging technologies for pointof care testing. Clin Biochem Rev 35(3): 155-167. 
8. Luppa PB, Müller C, Schlichtiger A, Schlebusch H (2011) Point-of- care testing (POCT): current techniques and future perspectives. TrAC Trends in Analytical Chemistry 30(6): 887-898.

9. O Kane MJ, McManus P, McGowan N, Lynch PL (2011) Quality error rates in point-of-care testing. Clin Chem 57(9): 1267-1277.
10. Callander S (2016) Point-of-care testing: ensuring patient safety through quality control. The Biomedical Scientist 60(2): 83-85.

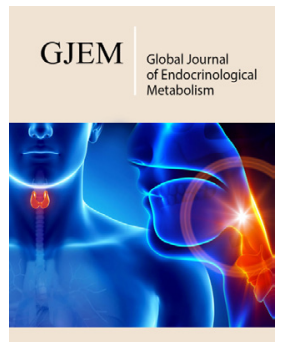

Global Journal of Endocrinological Metabolism

\section{Benefits of Publishing with us}

- High-level peer review and editorial services

- Freely accessible online immediately upon publication

- Authors retain the copyright to their work

- Licensing it under a Creative Commons license

- Visibility through different online platforms 